\title{
CORRECTION
}

\section{Correction to: Modeling habitat suitability for Yunnan Snub-nosed monkeys in Laojun Mountain National Park}

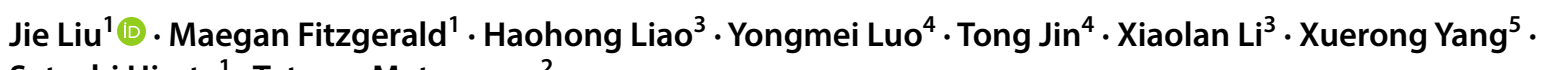
Satoshi Hirata ${ }^{1} \cdot$ Tetsuro Matsuzawa $^{2}$

Published online: 23 November 2019

(C) Japan Monkey Centre and Springer Japan KK, part of Springer Nature 2019

\section{Correction to: Primates}

https://doi.org/10.1007/s10329-019-00767-4

The correct name of the last author should be "Tetsuro Matsuzawa", and not "Tetsuro Matsuzwa" as given in the original publication of the article.

The original article has been corrected.

The original article can be found online at https://doi.org/10.1007/ s10329-019-00767-4.

Jie Liu

liujie871019@gmail.com

$\triangle$ Haohong Liao

haohong.liao@tnc.org

1 Wildlife Research Center, Kyoto University, Kyoto, Japan

2 Kyoto University Institute for Advanced Study, Kyoto, Japan

3 The Nature Conservancy, Lijiang, Yunnan, China

4 The Nature Conservancy, Beijing, China

5 Lijiang Laojun Mountain Biodiversity Conservation Center, Lijiang, Yunnan, China 